\title{
PRESERVAÇÃO DOS STAVES DOS ALTOS FORNOS THYSSENKRUPP CSA *
}

\author{
Alexandre Patrício Vieira da Silveira ${ }^{1}$ \\ Dalton Martins Neto ${ }^{2}$ \\ Eustáquio Vieira Junior ${ }^{3}$ \\ Sandro Valério Maia Larrubia ${ }^{4}$ \\ Felipe da Costa Oliveira ${ }^{5}$ \\ Alexandre da Silva Damasio 6
}

\section{Resumo}

A thyssenkrupp CSA (TKCSA), Rio de Janeiro, possui dois Altos-Fornos. Ambos iniciaram operação em 2010, Alto-Forno 01 em 13/07/2010 e Alto-Forno 02 em 06/12/2010. Em Dezembro de 2014, foram verificados problemas de deslocamento dos staves de ferro fundido e deformação dos staves de cobre. Analisando estes eventos foi identificado quebra dos parafusos de fixação dos staves de ferro fundido na carcaça. O trabalho apresenta histórico destes acontecimentos, as intervenções realizadas como meio de preservar a integridade e prolongar a vida útil dos Altos-Fornos e os resultados obtidos após retirada de água do interior do mesmo.

Palavras-chave: Alto-Forno; Stave; Parafuso de Fixação.

\section{Abstract}

\section{PRESERVATION OF STAVES}

The Thyssenkrupp CSA, Rio de Janeiro, has two Blast Furnaces. Both started the operations in 2010, Blast Furnace\#01 in 13/07/2010 and Blast Furnace\#02 in 06/12/2010. In December 2014, are showed displacement of the cast iron staves, and deformation in cooper stave. These displacements were identified by inspections on the stave compensators, caused by the brokes of the holding screws in the shell. The report presents the list of these hapennings, the actions realized to preserve the integrity and postpone the campaing of Blast Furnace, the planning and the results obtained after blocked the water into the equipament.

Keywords: Blast Furnace; Stave; Holding screws.

1 Técnico Especialista de Operações, Gerência de Alto-Forno, TKCSA - thyssenKrupp, Rio de Janeiro, Brasil.

2 Engenheiro Mecânico, Gerência de Alto-Forno, TKCSA - thyssenKrupp, Rio de Janeiro, Brasil.

3 Engenheiro Metalurgista, Gerência de Alto-Forno, TKCSA - thyssenKrupp, Rio de Janeiro, Brasil.

4 Engenheiro de Produção, Gerência de Alto-Forno, TKCSA - thyssenKrupp, Rio de Janeiro, Brasil.

5 Técnico de Processos, Gerência de Alto-Forno, TKCSA - thyssenKrupp, Rio de Janeiro, Brasil.

6 Técnico Mecânico, Gerência de Alto-Forno, TKCSA - thyssenKrupp, Rio de Janeiro, Brasil. 


\section{INTRODUÇÃO}

A Companhia Siderúrgica do Atlântico TKCSA possui dois Altos-Fornos [1] idênticos. Ambos iniciaram operação em 2010 sendo Alto-Forno 01 em 13/07/2010 e Alto-Forno 02 em 06/12/2010. Os Altos-Fornos da TKCSA possuem oito fileiras de staves sendo quatro fileiras de cobre e quatro fileiras de ferro fundido.

Em Julho de 2016 o Alto-Forno 02 apresentava grande instabilidade operacional e diminuição da temperatura do centro do cadinho. Essa irregularidade operacional foi causada por entrada de água no interior do forno, devido vários tubos de refrigeração dos staves estarem quebrados. Os tubos quebrados estavam fora do sistema principal, by-passados, sendo refrigerados por água industrial. Mesmo nessa condição havia entrada de 80.000 litros de água por dia para o interior do Alto-Forno 02. Diante deste problema foi realizado um exaustivo trabalho de recuperação das linhas de stave que estavam quebradas e de retirada de toda entrada de água para o interior do Alto-Forno 02.

Neste trabalho será apresentado o histórico das ocorrências no Alto-Forno 02 e as atividades realizadas para aumentar a vida útil dos staves.

As principais características dos Altos-Fornos são mostradas na tabela 1 e os resultados operacionais são mostrados na tabela 2

Tabela 1. Principais características dos Altos-Fornos TKCSA

\begin{tabular}{c|c|c}
\hline Itens & Unidade & AF's \\
\hline Volume interno & $\mathrm{m}^{3}$ & 3284 \\
\hline Volume de trabalho & $\mathrm{m}^{3}$ & 2775 \\
\hline Diâmetro do cadinho & $\mathrm{m}$ & 12,0 \\
\hline Capacidade de produção & $\mathrm{t} / \mathrm{dia}$ & 7500 \\
\hline Número de ventaneiras & - & 32 \\
\hline Número de furo de gusa & - & 2 \\
\hline Topo & - & $\begin{array}{c}\text { Topo sem } \\
\text { cone }\end{array}$ \\
\hline Sistema de granulação & - & INBA \\
\hline $\begin{array}{c}\text { Temperatura máxima de sopro } \\
\text { Sistema de refrigeração cuba, } \\
\text { ventre e rampa }\end{array}$ & $-\mathrm{C}$ & 1250 \\
\hline $\begin{array}{c}\text { Sistema de refrigeração do } \\
\text { cadinho }\end{array}$ & - & Stave-coolers \\
\hline $\begin{array}{c}\text { Início da campanha atual Alto } \\
\text { Forno 01 }\end{array}$ & - & Jaqueta \\
\hline $\begin{array}{c}\text { Fnício da campanha atual Alto } \\
\text { Forno 02 }\end{array}$ & - & $06 / 12 / 2010$ \\
\hline
\end{tabular}


Tabela 2. Dados operacionais dos Altos-Fornos TKCSA

\begin{tabular}{|c|c|c|c|c|c|c|c|}
\hline \multicolumn{8}{|c|}{ Alto-Forno 01} \\
\hline & BLOW IN & PRODUTIVIDADE & $\mathrm{CR}$ & ÓLEO & $\mathrm{PCl}$ & GN & FR \\
\hline campanhas & Data & $t / d / m^{3}$ & \multicolumn{5}{|c|}{$\mathrm{kg} / \mathrm{t}$} \\
\hline $1^{\circ}$ & $12 / 07 / 2010$ & 1,7 & 378 & - & 128,5 & - & 507 \\
\hline \multicolumn{8}{|c|}{ Alto-Forno 02} \\
\hline & BLOW IN & PRODUTIVIDADE & $\mathrm{CR}$ & ÓLEO & $\mathrm{PCl}$ & GN & FR \\
\hline campanhas & Data & $t / d / m^{3}$ & \multicolumn{5}{|c|}{$\mathrm{kg} / \mathrm{t}$} \\
\hline $1^{\circ}$ & $06 / 12 / 2010$ & 1,6 & 418 & - & 106,7 & - & 525 \\
\hline
\end{tabular}

\section{MATERIAIS E MÉTODOS}

\subsection{Reestabelecimento da refrigeração dos staves}

Em julho de 2016 após inspeções de rotina foi identificado que os tubos de refrigeração dos staves estavam se deslocando em movimentos radiais e axiais causando sua ruptura e possibilitando a entrada de água no interior do Alto-Forno 02. Como forma de minimizar a entrada de água, as linhas de refrigeração com tubo quebrado eram isoladas do circuito principal e instalado refrigeração com água industrial de baixa pressão. Este método não era efetivo, pois ainda permitia a entrada de água para o interior do forno. Após análise de causa, foi identificado que essa movimentação estava ocorrendo devido os parafusos de sustentação e travamento dos staves estarem quebrados. Essa quebra foi causada por possível sobre torque no parafuso de fixação e variações operacionais no processo.

Conforme verificado que a falta de fixação dos staves estava ocasionando ruptura dos tubos de refrigeração, foi desenvolvido formas de travamento dos mesmos. Para staves de ferro fundido criou-se o procedimento de fixar pelo próprio tubo, utilizando um anel de aço soldado no próprio tubo de refrigeração e um compensador modificado. Para reparo dos staves de cobre foi desenvolvido forma de refazer a furação da carcaça para realinhamento dos tubos. Em situações onde haviam tubos quebrados os mesmos foram recuperados através de procedimento de soldagem, que consiste em prolongar o tubo utilizando luva de aço. Em casos onde não era possível a soldagem, foi inserido um tubo flexível para reestabelecer à refrigeração. Casos onde há queima do flexível temos como opção instalação de placas de refrigeração (cigar cooler).

As etapas de reparo serão mostradas a seguir.

\subsection{Planejamento do reparo por soldagem}

A forma utilizada para reestabelecer a refrigeração do stave de ferro fundido por soldagem consiste nos seguintes passos:

- Retirar a água de refrigeração para liberar tubulação para corte com maçarico 
- Cortar o compensador da linha danificada

- Cortar o tubo de refrigeração

- Preparar as superfícies do tubo e da parede

- Soldar a luva de prolongamento do tubo quando necessário

- Soldar o tubo, reconstituindo a linha de refrigeração.

- Realizar testes no tubo: hidrostático e liquido penetrante (LP)

- Soldar a arruela e realizar teste de LP

- Soldar compensador e realizar teste de LP

As principais etapas são mostradas na figura 01 .

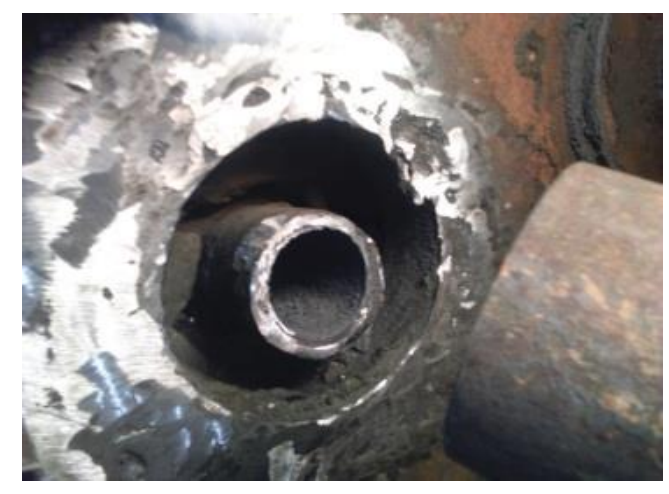

a) Etapa de corte do tubo

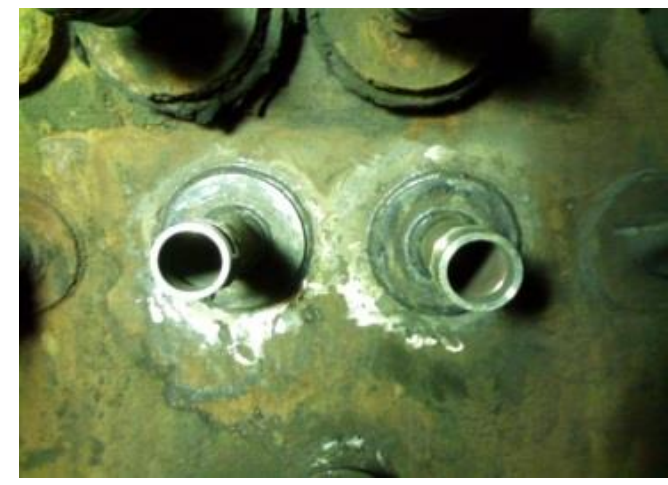

c) Etapa de soldagem do tubo

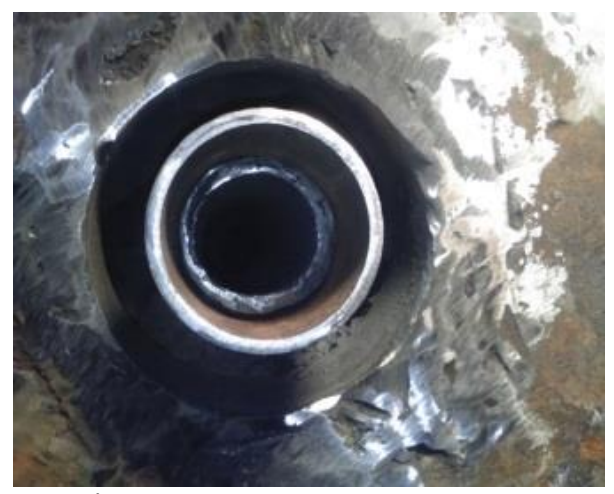

b) Etapa de soldagem da luva

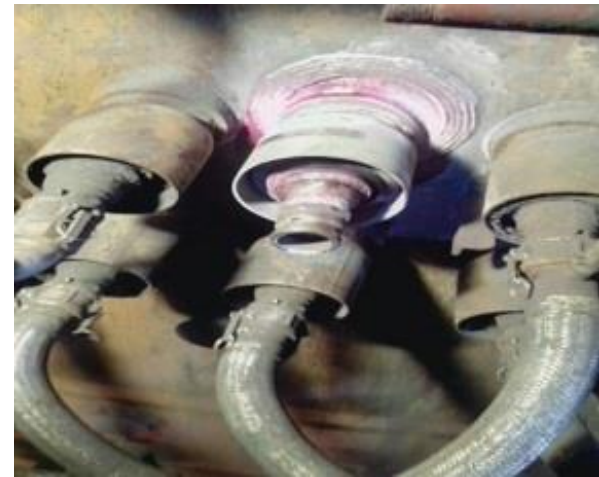

d) Etapa de soldagem do compensador

Figura 1. Etapas do processo de reparo por soldagem

\subsection{Planejamento de reparo por inserção de flexível}

Em casos onde não é possível o reparo do tubo por solda é feita a instalação de flexível no interior da linha conforme os passos a seguir:

- Cortar a tubulação de 2" de entrada do staves, com 100mm de comprimento

- Preparar tubulação para receber engate rápido pino macho e soldá-lo na tubulação

- Passar o cabo de aço na tubulação especificada do stave

- Passar o flexível de 1" no interior do dispositivo engate rápido + redução 2" $x$ 1 1/2" (este será p/ a saída

- Passar o flexível dentro da tubulação especificada do stave e soldar engate macho de 2"

- Conectar engate rápido na tubulação do stave - ponta superior e inferior

- Restabelecer refrigeração na linha com stave 
- Conectar engate rápido na tubulação do stave - ponta superior

- Soldar na redução (1 1/2") a conexão do flexível - ponta superior

- Realizar teste de lp nas soldas e corrigir não conformidades

- Instalar niple de redução 1" x 1/2" + válvula de 1/2" + espigão 3/4" na conexão do flexível e conectar mangueira de 3/4" (4 metros)

- Passar o flexível de 1" no interior do dispositivo engate rápido + redução ponta inferior

- Marcar e cortar flexível no tamanho ideal para instalação das conexões,

- Soldar luva específica na ponta do flexível

- Soldar flexível Soldar adaptador de 1" na ponta do conjunto

- Realizar teste de LP nas soldas e corrigir não conformidades

- Instalar niple 1" + válvula de 1" + redução de 2" x 1" e engate rápido macho na conexão do flexível

- Instalar niples e válvulas de 1/2" para injeção de massa

- Teste hidrostático

- Injeção de massa

- Liberação

As figura 02 mostra os detalhes deste tipo de reparo.

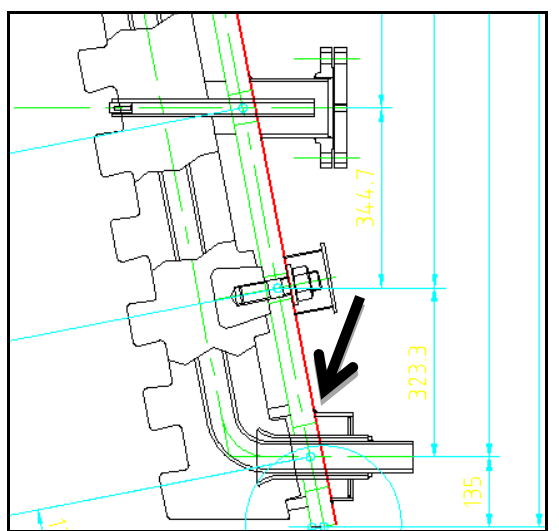

a) Cortar a tubulação de 2" de entrada do staves, com $100 \mathrm{~mm}$ de comprimento

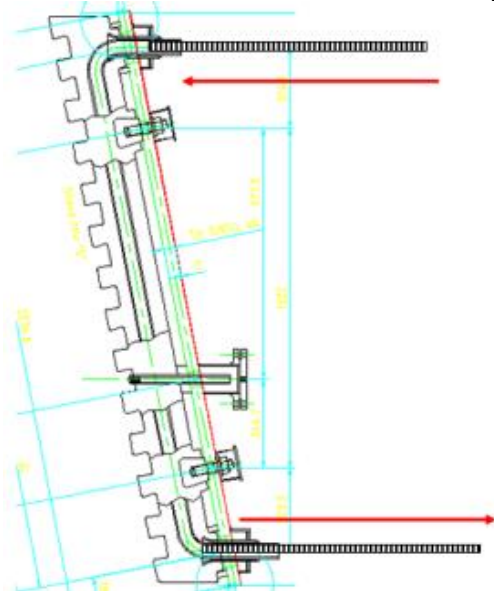

c) Passar o flexível dentro da tubulação especificada do stave e soldar no engate macho de 2"

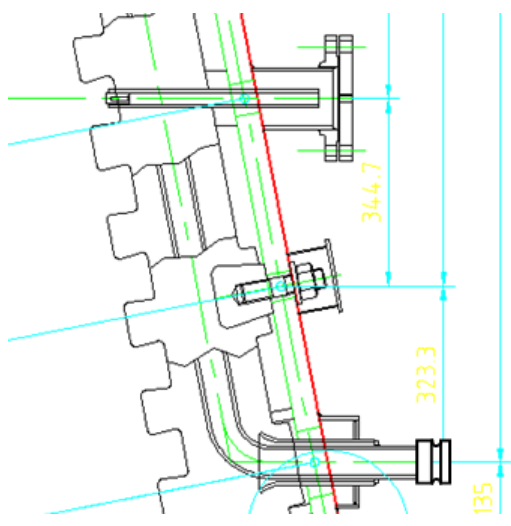

b) Preparar tubulação para receber engate rápido pino macho e soldá-lo na tubulação;

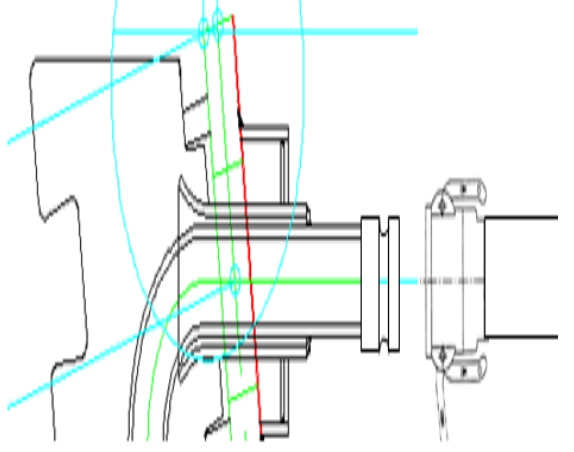

d) Reconectar mangote de stave e normalizar refrigeração

Figura 2. Principais etapas do processo de reparo por flexível 


\subsection{Planejamento de reparo por placa ou (cigar cooler)}

Quando ocorre a queima do flexível é feita a instalação de cigar cooler ou placa de refrigeração.

O passo a passo para a montagem é descrito abaixo:

- Isolar a linha com massa refratária

- Furar a carcaça com serra copo

- Preparar a superfície

- Inserir o cigar ou placa

- Soldar luva de aço na carcaça

- Fazer procedimento de LP

- Reestabelecer refrigeração

- Liberar para operação

A figura 03 mostra preparação e este tipo de refrigeração instalado.

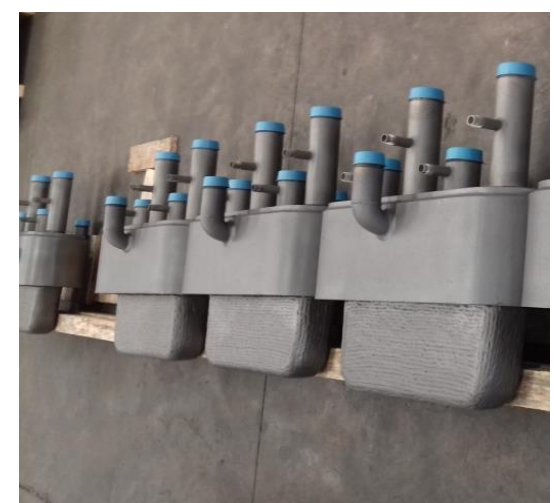

a) Placas de refrigeração

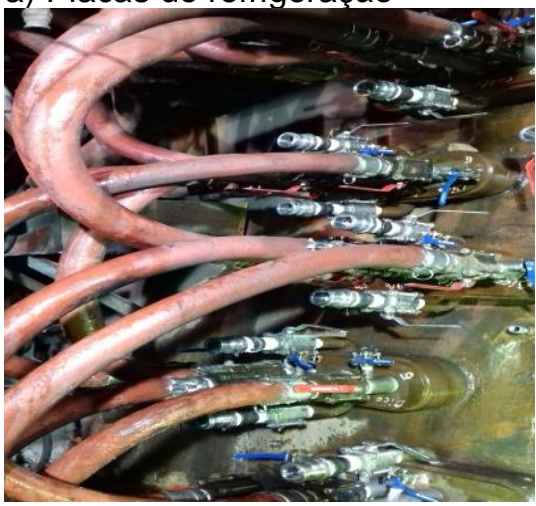

c) Placas e cigar instalado

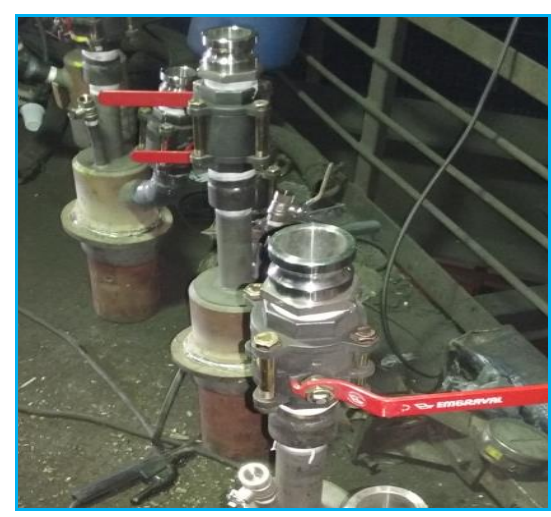

b) Cigar cooler

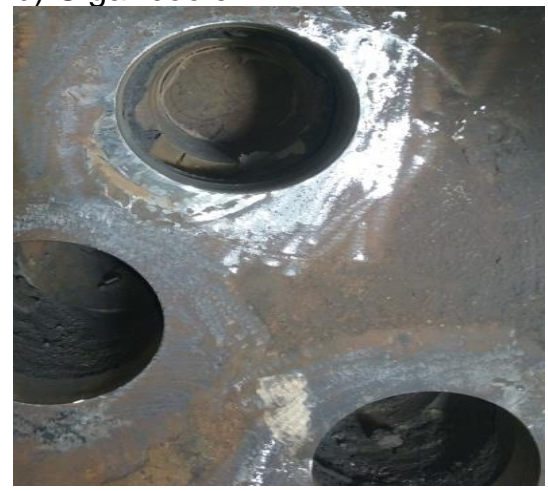

d) Furo na carcaça para cigar

Figura 3. Instalação de placas e cigar cooler na carcaça do Alto-Forno

\subsection{Realinhamento do tubo do stave de cobre}

Quando ocorre o deslocamento do tubo do stave de cobre em relação carcaça é feito a correção através de realinhamento conforme descrito nos seguintes passos

- Retirar compensador antigo 
- Efetuar limpeza da carcaça

- Cortar trecho da carcaça no diâmetro de $160 \mathrm{~mm}$

- Reposicionar trecho da carcaça alinhando com o tubo de refrigeração do stave

- Soldar trecho da carcaça e fazer teste de líquido penetrante

- Soldar novo compensador

- Liberar para operação

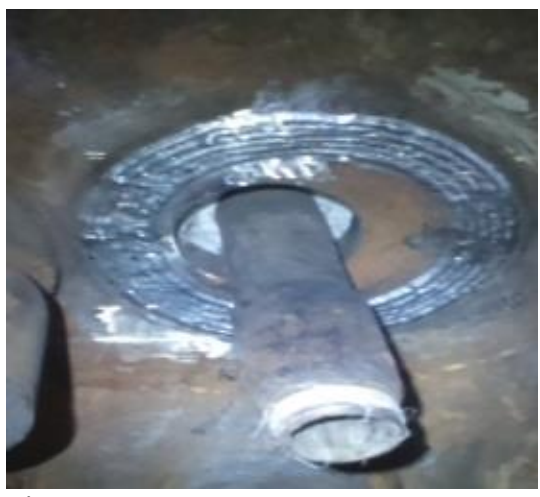

a) Tubo do stave de cobre realinhado

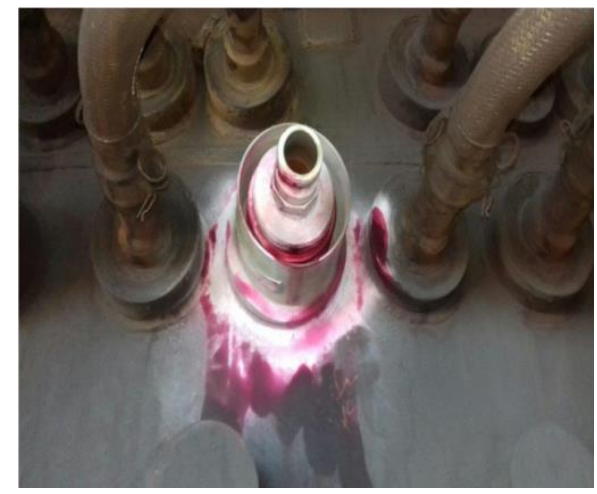

b) Compensador novo instalado

Figura 4. Realinhamento do tubo do stave de cobre na carcaça do Alto-Forno 


\section{RESULTADOS E DISCUSSÃO}

\subsection{Resultados Operacionais Alto-Forno 02 Após Reparo}

O gráfico da figura 5 evidencia que a retirada de água no interior do Alto-Forno 02 no ano de 2016 foi fundamental para melhora da permeabilidade e reativação do cadinho. O gráfico da figura 06 mostra a quantidade de linhas com água no AltoForno 02

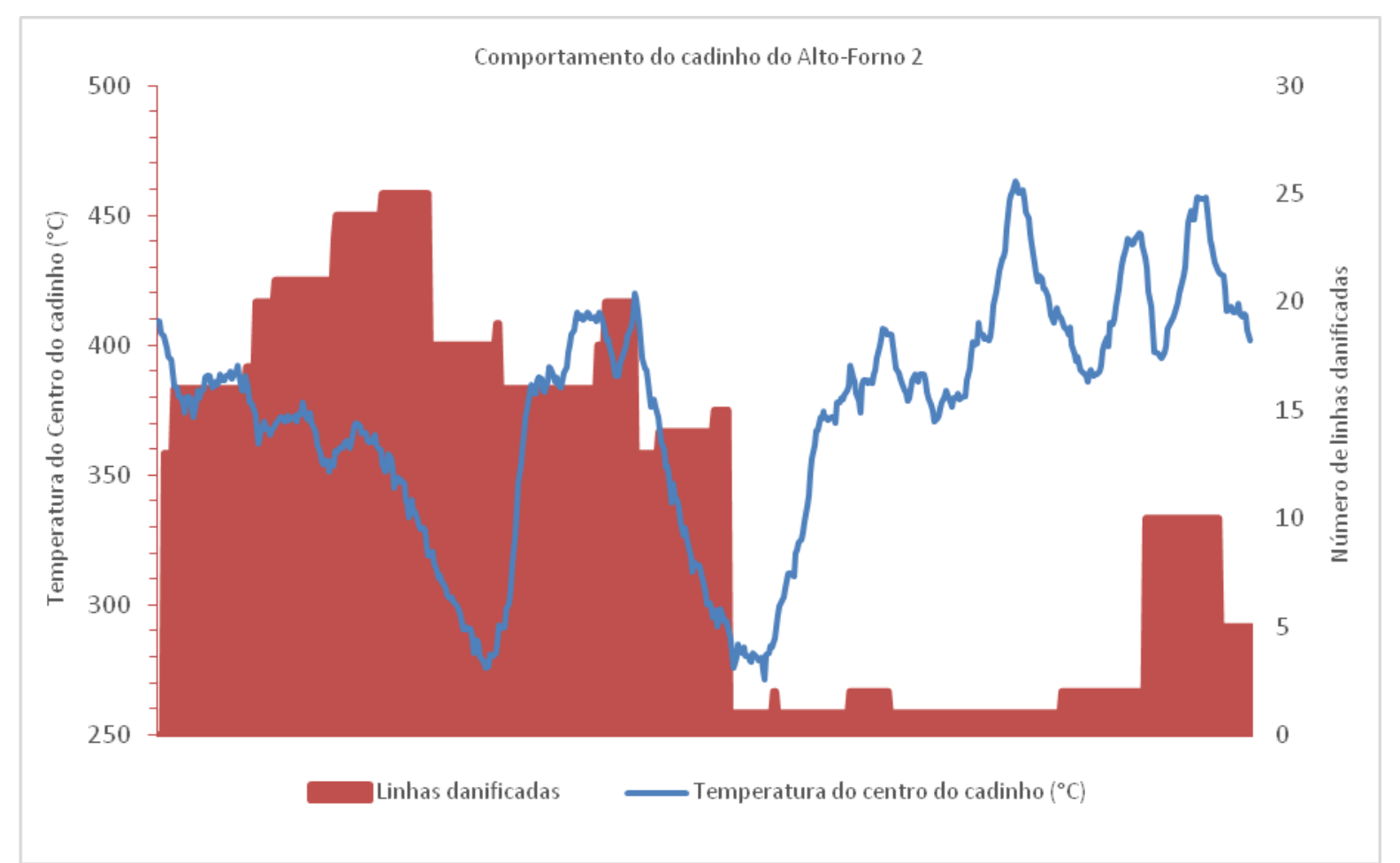

Figura 5. Influência da retirada de água do Alto-Forno 02 para ativação do cadinho

\section{Overview staves situation BF2}

Figura 6 Linhas com água industrial Alto-Forno 02 


\section{CONCLUSÃO}

Todo o trabalho de recuperação das linhas de stave no Alto-Forno 02 vem rendendo bons resultados e deve acontecer de forma continua, pois tem influência direta na operação do Alto-Forno 02. Dessa forma conseguiu-se obter bons resultados tornando o processo mais estável devido melhor permebealização do cadinho.

\section{REFERÊNCIAS}

1 BABICH, A.; SENK, D.; GUDENAU,H.W.;MAVROMMATIS.K.TH. Ironmaking Textbook - Aachen: RWTH; 2008. 